\title{
Assessment of $F_{200}$ fluence for major solar energetic particle events on the multi-millennial time scale
}

\author{
Ilya G. Usoskin* ${ }^{* \dagger}$ \\ Sodankylä Geophysical Observatory and ReSoLVE Center of Excellence, University of Oulu, \\ Finland \\ E-mail: Hlya.usoskindoulu.fi
}

\section{Gennady A. Kovaltsov}

Ioffe Physical-Technical Institute, St. Petersburg, Russia

E-mail: gen.kovalemail.ru

\section{Edward W. Cliver}

National Solar Observatory, Sunspot, NM 88349, USA

E-mail: ecliveranso.edu

\section{William F. Dietrich}

Consultant, Prospect Heights, IL 60070 USA

E-mail: dietrich.uchicagodgmail.com

\author{
Allan J. Tylka \\ Code 672, NASA Goddard Space Flight Center, Greenbelt, MD 20771, USA \\ E-mail: allan.j.tylkadnasa.gov
}

\begin{abstract}
Solar energetic particle (SEP) fluxes are typically quantified in the $F_{30}$ units (integrated fluence of particles with energy above $30 \mathrm{MeV}$ ) and their direct measurements are available only for the last several decades. On the other hand, a reconstruction of major SEP events in the distant past (centennial-millennial time scales) is possible using data on the cosmogenic isotopes such as ${ }^{1} 4 \mathrm{C}$ and ${ }^{10} \mathrm{Be}$ measured in stratified and independently datable terrestrial archives (tree trunks or polar ice cores). While such events (or at least candidates for those) can be identified in the cosmogenic records with a degree of confidence, the estimate of the $F_{30}$ flux/fluence is ambiguous depending on the proposed SEP energy spectrum. Here we introduce a new index, the integral fluence of an SEP above $200 \mathrm{MeV}, F_{200}$, which is related to the effective energy of the production of the cosmogenic isotopes by SEP in the Earth atmosphere. This index is robust against the assumptions on the exact shape of the energy spectrum of the event in a wide range of parameters. Using several records of cosmogenic isotopes, we present a reconstruction of the $F_{200}$ fluence for major SEP events in the past, and also assess the occurrence probability density function for extreme events. In particular, we evaluate that extreme SPEs with $F_{200}>10^{10} \mathrm{~cm}^{-2}$ may occur no more frequently than once per 10000 years or even rarer up to never.
\end{abstract}

The 34th International Cosmic Ray Conference,

30 July- 6 August, 2015

The Hague, The Netherlands 


\section{Introduction}

Solar energetic particle (SEP) events occur sporadically in association with eruptive phenomena (solar flares or coronal mass ejections) on the Sun. Such events are accompanied by greatly enhanced (by several orders of magnitude) flux of solar energetic particles (mostly protons), accelerated to high energy up to several $\mathrm{GeV}$ of kinetic energy in the solar corona or in the interplanetary space. Such enhanced fluxes can cause serious radiation hazards for human beings, in particular at transpolar jet flights and even more severe for astronauts in orbit. Hazards can be the critical level for the modern technological community, leading to malfunctioning or even damage of various communication and navigation systems, and they even may potentially affect Earth's climate and biosphere [ [ [ ] ]. Therefore, it crucially important, in particular for planning and designing of future missions, to have an assessment of possible radiation hazards [B]]. Our knowledge of the SEP events is limited to the decades, starting with the first detection of a very strong SEP event by ground based ionization chambers in 1942 [䧃], followed by ground-based neutron monitors since 1952 and extended by direct space-borne monitoring since the 1970s. Although quite a large number of SEP events have been recorded and studied, the statistics of very strong and extreme events is poor being limited to several events [3]. Moreover, the last decades, for which we have direct data on SEP events, were quite unusual being a part of the modern Grand maximum of solar activity [5] characterized by very active Sun, which may not be fully representative. This very active period is known to cease now so that we expect the qualitatively different level (low to moderate) of solar activity in the near future.

This is important for physics of solar flares as there is a correlation between fluxes of solar energetic particles and strength of solar eruptive events in the solar atmosphere [四].

For many purposes, particularly so for the radiation hazards, it is useful to quantify the strength of a SEP event by a single number. Considering the effective energy of SEP it is often taken as the fluence (the integral flux over the total duration of the event) of SEPs with kinetic energy above $30 \mathrm{MeV}$, conventionally called $F_{30}$ [B]]. Sometime also the fluences $F_{10}$ (SEPs with energy $>10$ $\mathrm{MeV})$ or $F_{60}(>60 \mathrm{MeV})$ are considered. These fluences are computed from direct space-borne measurements.

Accordingly, only indirect proxy data may help in reconstruction of the history of major SEP events in the past and thus lead to a quantitative assessment of the occurrence probability for such events. The usual proxy for the variability of cosmic rays and also for major SEP events is based on records of cosmogenic radionuclides, such as ${ }^{10} \mathrm{Be}$ measured in polar ice and ${ }^{14} \mathrm{C}$ measured in dendrochronologically dated tree rings $[\square,[]$. A short list of possible candidates for major SEP events in the past was proposed recently [Q] providing a statistic on the time scale of ten millennia. Two events have been confirmed as the events by high-resolution annual data of cosmogenic ra-

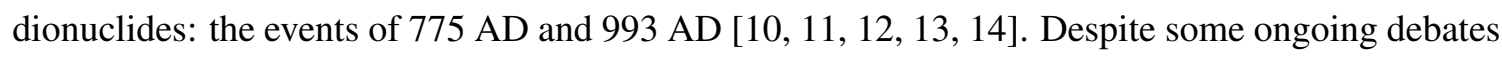
about the origin of these events [[5], [2, [6], solar origin of the spikes due to extreme SEP events is the most likely source, and the overall production of the ${ }^{14} \mathrm{C}$ and ${ }^{10} \mathrm{Be}$ nuclides can be calculated.

However, when using proxy data one cannot evaluate the spectrum of SEPs and have to make an assumption of that. Since the effective energy of cosmogenic isotope production in the atmo-

\footnotetext{
*Speaker.

${ }^{\dagger}$ A footnote may follow.
} 
sphere is relatively high [ [प, प]], the low energy part of the spectrum remains quite uncertain in this approach. Depending on whether the SEP spectrum is assumed to be hard or soft, the evaluation of the $F_{30}$ fluence from the reconstructed data can be uncertain with the factor up to 50 [Q, [2] which makes it difficult to link the reconstructions to the modern data.

Here we look for such a parameter of SEP spectra that can be directly assessed from the cosmogenic isotope data without any assumptions on the shape of the SEPs spectrum.

\section{Spectrum of SPEs}

Here we consider the spectral shape of the SEP event integral spectra in the form of the Band function [18] which describes the integral energy spectrum as a double power-law with a smooth exponential roll-over in-between. Both the function and its first derivative keep continuous through energy. Let us express rigidity $R$ in $\mathrm{GV}$ and kinetic energy $E$ in $\mathrm{GeV}$. Then the integral omnidirectional event-integrated fluence (in protons $/ \mathrm{cm}^{2}$ ) of SEP events can be represented as

$$
\begin{array}{lr}
F(>E)=J_{0} \cdot R^{-\gamma_{1}} \exp \left(-R / R_{0}\right), & \text { for } R \leq R_{\mathrm{b}}, \\
F(>E)=J_{0} \cdot A \cdot R^{-\gamma_{2}}, & \text { for } R>R_{\mathrm{b}},
\end{array}
$$

where

$$
\begin{aligned}
& A=\left[R_{\mathrm{b}}\right]^{\left(\gamma_{2}-\gamma_{1}\right)} \exp \left(\gamma_{1}-\gamma_{2}\right), \\
& R=\sqrt{E^{2}+2 E_{0} \cdot E},
\end{aligned}
$$

where $R_{\mathrm{b}}=\left(\gamma_{2}-\gamma_{1}\right) R_{0}$ and $E_{0}=0.938 \mathrm{GeV}$ is the proton's rest-mass energy. Throughout the paper we will call $F(>E)$ as $F_{E}$ where $E$ is expressed in $\mathrm{MeV}$ (e.g., $\left.F(>200 \mathrm{MeV}) \equiv F_{200}\right)$. We note that the spectral index $\gamma_{2}$ describes the high-energy tail of the spectrum that we will use below to quantify the hardness of the spectrum.

Integral spectra (spectral fluences) have been evaluated [ए8] for all major ground level enhancement (GLE) events for the last decades. The spectra vary essentially between the events as illustrated in Fig. W which shows the ratio of the $F_{\text {En }}\left(>\mathrm{E}_{\mathrm{n}}\right)$ to that $F_{200}$ for three most interesting GLE events. One is GLE \#5 (see the official numbering of the GLE events at http://GLE.oulu.fi) which took place on 23-Feb-1956 and was the strongest known GLE event with a very hard spectrum - this can be observed as an 'elevated' high-energy tail. The GLE \#10 event (12-Nov-1960) was a typical GLE event with the spectrum close to the mean spectrum over all the events. The GLE \#24 event (04-Aug-1972) was a relatively weak GLE with a soft spectrum but it was the greatest even in the sense of $F_{30}$ fluence. While upper and former cases bound all known GLE spectral fluences, the middle one represents a typical spectrum. The $F_{200}$ fluence for the three GLE events discussed above was $1.2 \times 10^{8}, 6.4 \times 10^{7}$ and $1.4 \times 10^{7}$ protons $/ \mathrm{cm}^{2}$, for the events of $23-\mathrm{Feb}-1956$, 12-Nov-1960 and 04-Aug-1972, respectively.

\section{Production of cosmogenic isotopes during SEP events}

\subsection{Cosmogenic ${ }^{14} \mathrm{C}$}

We have calculated the global production of ${ }^{14} \mathrm{C}, Q_{14 \mathrm{C}}$, by a SEP event in the Earth's atmosphere, using a recent production model based on a full Monte-Carlo simulation of the cosmic-ray 


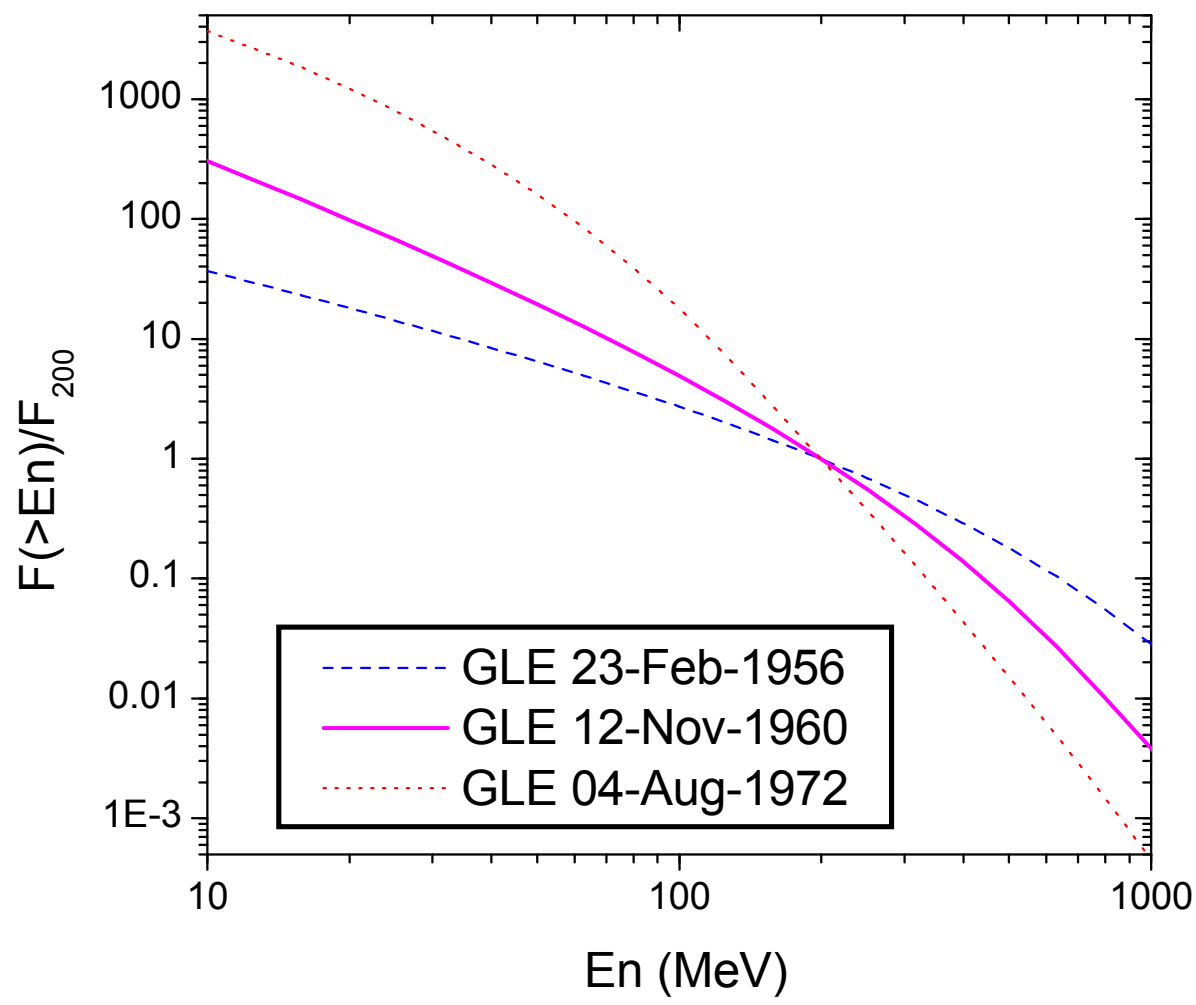

Figure 1: The ratio of the integral fluence of SEP $\left(F\left(>E_{\mathrm{n}}\right)\right)$ to $F_{200}$, which is normalized to unity for $E_{\mathrm{n}}=200 \mathrm{MeV}$. Different curves correspond to three GLE events, as denoted in the legend: GLE \#5 (23Feb-1956), GLE \# 10 (12-Nov-1960) and GLE \#24 (04-Aug-1972).

induced atmospheric cascade [20]. The computations were performed for different SEP integral spectra (see Fig. W). In order to estimate the effect of the spectral uncertainties, we have have normalized the computed values of $Q_{14 \mathrm{C}}$ are normalized to the fluences $F_{E n}$ for each scenario. We have calculated the yield (the number of produced ${ }^{14} \mathrm{C}$ atoms $/ \mathrm{cm}^{2}$ averaged over the globe) by SEP protons with the unit fluence with energy above $E_{\mathrm{n}}, Y=Q / F_{E n}$. It is depicted in Figure QA. One can see that the spread of the yield is large for $E_{\mathrm{n}}=30 \mathrm{MeV}$, being a factor of 40 between soft and hard spectra. This implies that the estimate of $F_{30}$ is ambigous for the same ${ }^{14} \mathrm{C}$ production may vary by orders of magnitude depending on the assumes spectrum of SEPs, so that it is greater for soft spectra and smaller for hard spectra. The too high reference energy, e.g. $E_{\mathrm{n}}=1000 \mathrm{MeV}$ would also lead to a huge (two orders of magnitude) ambiguity of the fluence but in the opposite direction.

On the other hand, one can see that the choice of the reference energy $E_{\mathrm{n}}=200 \mathrm{MeV}$ leads to a very stable (within 30\%) link between the ${ }^{14} \mathrm{C}$ production and the $F_{200}$ fluence irrespectively of the spectral shape of the SEP event. This implies that $F_{200}$ is nearly directly related to the SEP-related atmospheric production of ${ }^{14} \mathrm{C}$. Within the reasonable range of the SEP integral fluences (Fig. W) one can estimate that $(1.6 \pm 0.2) \times 10^{-2}$ atoms $/ \mathrm{cm}^{2}$ of globally averaged ${ }^{14} \mathrm{C}$ is produced in the Earth's atmosphere for the unit $F_{200}$ fluence. Thus, the measured increase of ${ }^{14} \mathrm{C}$ due to SEP event can be directly translated into the $F_{200}$ fluence without uncertainties related to the exact spectral shape, in contrast to the lower-energy fluence $F_{30}$. 


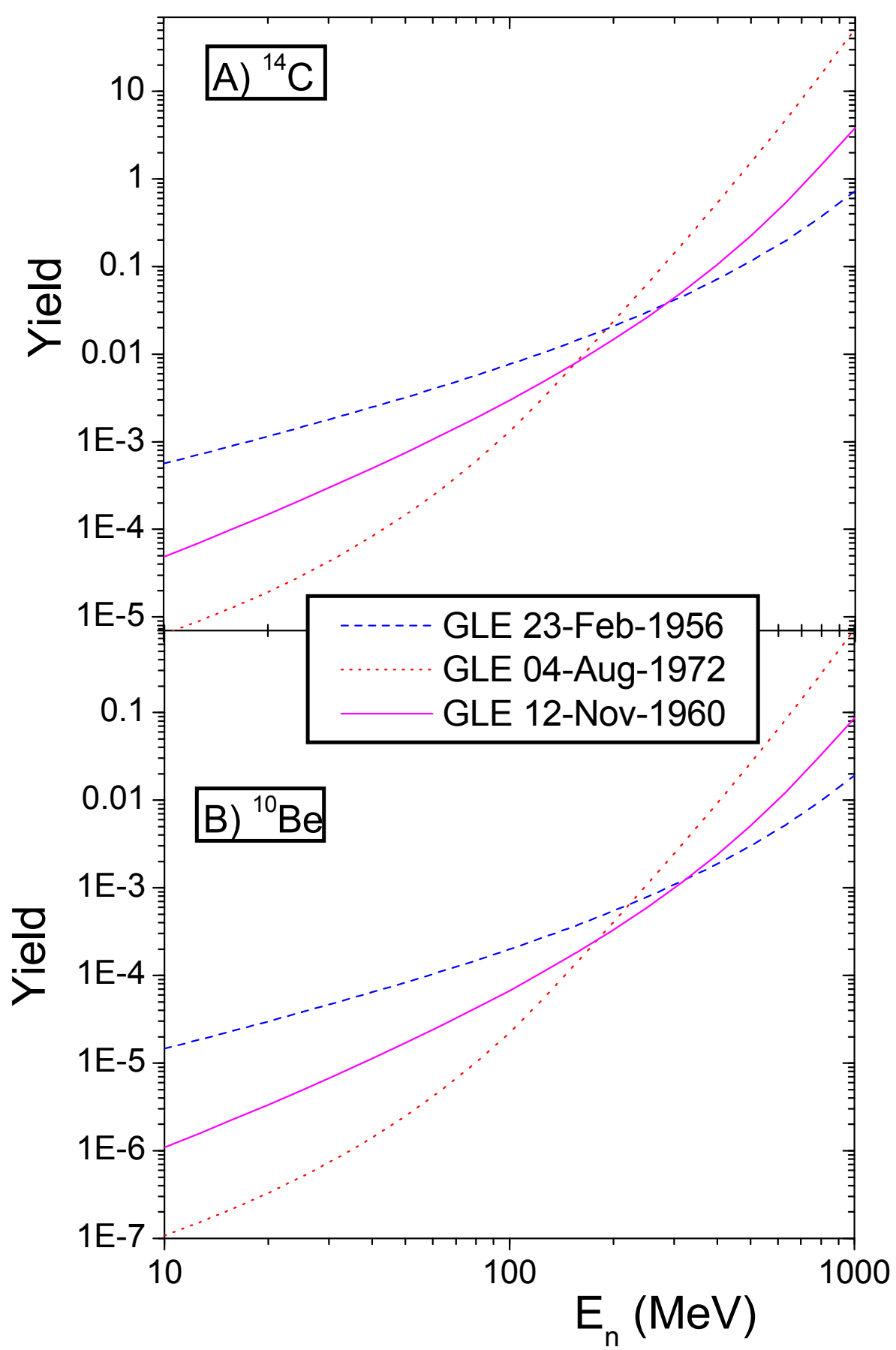

Figure 2: Yield (production per unit fluence of SEPs) of cosmogenic isotopes for integral fluences shown in Fig. 1 as function of the event-integrated fluence of $\operatorname{SEP}\left(F_{\mathrm{En}}\right)$. Panel A) Modelled yield curves for ${ }^{14} \mathrm{C}$ per one solar energetic proton with energy above $E_{\mathrm{n}}$. Panel B) Modelled yield curves for ${ }^{10} \mathrm{Be}$ per one solar energetic proton with energy above $E_{\mathrm{n}}$. (Modified after ref. [एप].) 
Using this relation we can estimate the values of $F_{200}$ for the events of $775 \mathrm{AD}$ and $993 \mathrm{AD}$ as $\approx 8 \times 10^{9}$ and $6 \times 10^{9} \mathrm{~cm}^{-2}$, respectively, which is 66 and 50 times greater, respectively, than the largest event of the space era, on 23-Feb-1956.

We also estimated $F_{200}$ for the Carrington event of $1859 \mathrm{AD}[\mathbb{[ 2 ]}]$ based on annual ${ }^{14} \mathrm{C}$ data [22, [23] for that period. Since there is no clear enhancement of $\Delta^{14} \mathrm{C}$ greater than 2 permille (which corresponds to measurement errors), a conservative upper limit for the Carrington event is $F_{200} \leq 10^{9} \mathrm{~cm}^{-2}$.

\subsection{Cosmogenic ${ }^{10} \mathrm{Be}$}

Similarly we analyzed the relation between the SEP event fluence and production and transport of ${ }^{10} \mathrm{Be}$. Here we applied a recent model of ${ }^{10} \mathrm{Be}$ production [24] and computed deposition of ${ }^{10} \mathrm{Be}$ in a $3 \mathrm{D}$ realistic atmosphere, assuming the polar tropospheric and hemispherically mixed stratospheric productions (see, e.g., [25, [26]). The yield of ${ }^{10} \mathrm{Be}$ computed in this way and deposited in polar ice is shown in Figure $\square \mathrm{B}$. One can see that the relation between the fluence $F_{E n}$ and the ${ }^{10} \mathrm{Be}$ deposition is optimal at $E_{\mathrm{n}} \approx 200 \mathrm{MeV}$. At this energy, the uncertainty of the $F_{200}$ related to the unknown spectral shape is minimum and does not exceed $30 \%$, similarly to ${ }^{14} \mathrm{C}$. Thus, one can evaluate that the deposition of ${ }^{10} \mathrm{Be}$ in polar ice is $(3.4 \pm 0.5) \cdot 10^{-4} \mathrm{~cm}^{-2}$ for the unit $F_{200}$ fluence .

\section{Evaluation of $F_{200}$ for different events}

The value of $F_{200}$ for the known GLE events for the space era since the 1950s reaches up to $1.2 \times 10^{8}$ protons $/ \mathrm{cm}^{2}[\mathbb{Q}]$ ]. The cumulative occurrence probability density function of the annual values of $F_{200}$ is presented in Figure [3. The corresponding values are presented in Fig. [ 3 as open triangles. Of special interest is to assess strongest events in the past [ $\left[\right.$ ]]. We have computed the $F_{200}$ fluence for the events listed as candidates of extreme SEP events over the Holocene [9, 미, ㅁ]].

It implies a strong roll-over of the events with an extreme fluence (cf. [27, 6]), which means that the Sun has less probability to produce extreme events than I might though based only on the space era data. The steepening of the distribution appears somewhere around $F_{200}=10^{9} \mathrm{~cm}^{-2}$. The gap between low- and high-fluence branches of the distribution is likely caused by the detection threshold of SEP events using cosmogenic isotopes method. Alternatively, we may be looking at different populations of events. This result suggests that the strongest, in the sense of the $F_{200}$ values, events (the $775 \mathrm{AD}$ event [एU]], may be $\approx 10-50$ times stronger than those for the strongest recent cycle.

\section{Conclusions}

We have proposed a new index of the strength of solar energetic particle events, which is related to the event-integrated fluence of protons above $200 \mathrm{MeV}, F_{200}$. This $F_{200}$ fluence is useful since it is related to production of cosmogenic isotopes ${ }^{14} \mathrm{C}$ and ${ }^{10} \mathrm{Be}$ in the Earth's atmosphere, independently on the assumptions of the energy spectrum of SEPs during the event. Therefore, the value of the $F_{200}$ fluence can be directly evaluated from the cosmogenic isotope data in the past, while the conventional $F_{30}$ fluence has huge uncertainty in this kind of reconstruction up to a factor of 40 [एव]. We have performed an estimate of the cumulative occurrence probability density 


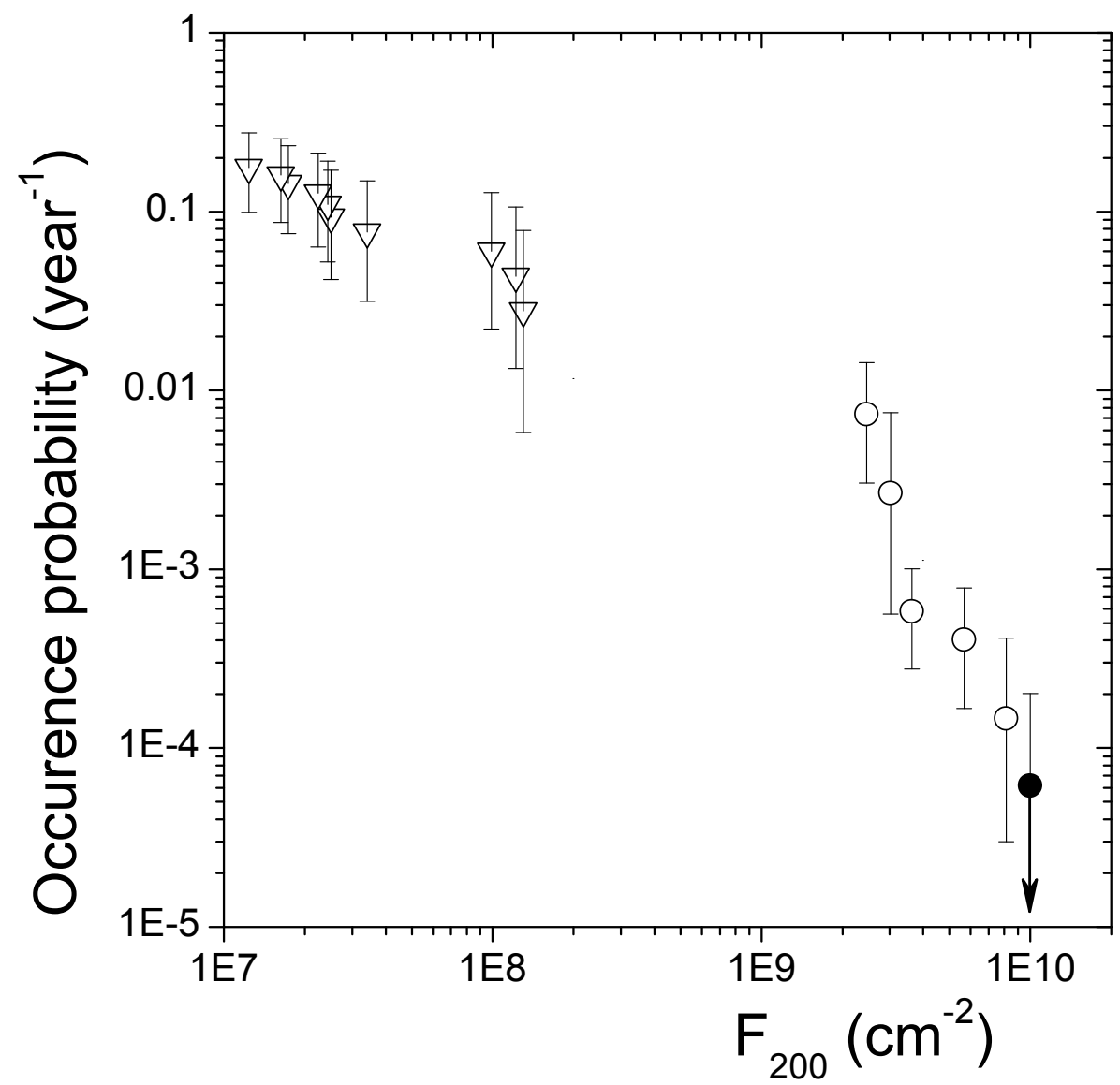

Figure 3: The cumulative occurrence probability density function of annual $F_{200}$ fluence of SEP. Panel (a) is for the $F_{200}$ obtained here. The triangles represent fluences for the GLE events measured during the space era, while open circles correspond to the events evaluated from cosmogenic isotope data in the past. The filled circle denotes a conservative upper limit (no events with the $F_{200}$ fluence greater than $10^{10} \mathrm{~cm}^{-2}$ have been observed during 11000 years). Error bars correspond to the $90 \%$ confidence level evaluated assuming Poisson statistics. (Modified after ref. [एप]].

function for extreme SEP events using data from both direct measurements during the modern space-era epoch and indirect proxy of the cosmogenic isotope data for the Holocene. We estimate that no extreme SEP events with the $F_{200}$ fluence greater than $10^{10} \mathrm{~cm}^{-2}$ occur more frequent than once per 10-15 millennia.

It is important to provide new data series with high (annual) resolution for the whole Holocene of cosmogenic isotopes in order to obtain better assessments of the SEP fluence in the range of $F_{200}$ values between $10^{8}$ and $10^{9} \mathrm{~cm}^{-2}$.

\section{Acknowledgements}

IU's contribution was done in the framework of the ReSoLVE Centre of Excellence (Academy of Finland, project no. 272157). AJT and WFD are supported by NASA Solar \& Heliophysics SR\&T under NNH12AT09I. 


\section{References}

[1] R. Vainio, L. Desorgher, D. Heynderickx, M. Storini, E. Flückiger, R. B. Horne, G. A. Kovaltsov, K. Kudela, M. Laurenza, S. McKenna-Lawlor, H. Rothkaehl, and I. G. Usoskin, Dynamics of the Earth's particle radiation environment, Space Sci. Rev. 147 (2009) 187-231.

[2] D. Atri and A. L. Melott, Cosmic rays and terrestrial life: A brief review, Astropart. Phys. 53 (2014) 186-190, [arXiv:121].3962]].

[3] M. A. Shea and D. F. Smart, Space Weather and the Ground-Level Solar Proton Events of the 23rd Solar Cycle, Space Sci. Rev. 171 (2012) 161-188.

[4] S. E. Forbush, Three Unusual Cosmic-Ray Increases Possibly Due to Charged Particles from the Sun, Phys. Rev. 70 (1946) 771-772.

[5] S. Solanki, I. Usoskin, B. Kromer, M. Schüssler, and J. Beer, Unusual activity of the sun during recent decades compared to the previous 11,000 years, Nature 431 (2004) 1084-1087.

[6] C. J. Schrijver, J. Beer, U. Baltensperger, E. Cliver, M. Güdel, H. Hudson, K. McCracken, R. Osten, T. Peter, D. Soderblom, I. Usoskin, and E. Wolff, Estimating the frequency of extremely energetic solar events, based on solar, stellar, lunar, and terrestrial records, J. Geophys. Res. 117 (2012) A08103.

[7] I. Usoskin, S. Solanki, G. Kovaltsov, J. Beer, and B. Kromer, Solar proton events in cosmogenic isotope data, Geophys. Res. Lett. 33 (2006) L08107.

[8] W. Webber, P. Higbie, and K. McCracken, Production of the cosmogenic isotopes ${ }^{3} h,{ }^{7}$ be, ${ }^{10}$ be, and ${ }^{36} \mathrm{cl}$ in the earth's atmosphere by solar and galactic cosmic rays, J. Geophys. Res. 112 (2007).

[9] I. G. Usoskin and G. A. Kovaltsov, Occurrence of Extreme Solar Particle Events: Assessment from Historical Proxy Data, Astrophys. J. 757 (2012) 92, [arxiv:1207.5932].

[10] F. Miyake, K. Nagaya, K. Masuda, and T. Nakamura, A signature of cosmic-ray increase in ad $774 U ̈ 775$ from tree rings in Japan, Nature 486 (2012) 240-242.

[11] F. Miyake, K. Masuda, and T. Nakamura, Another rapid event in the carbon-14 content of tree rings, Nature Comm. 4 (2013).

[12] I. G. Usoskin, B. Kromer, F. Ludlow, J. Beer, M. Friedrich, G. A. Kovaltsov, S. K. Solanki, and L. Wacker, The AD775 cosmic event revisited: the Sun is to blame, Astron. Astrophys. 552 (2013) L3, [arxiv: [302,689]].

[13] A. J. T. Jull, I. P. Panyushkina, T. E. Lange, V. V. Kukarskih, V. S. Myglan, K. J. Clark, M. W. Salzer, G. S. Burr, and S. W. Leavitt, Excursions in the 14 c record at ad 774-775 in tree rings from russia and america, Geophys. Res. Lett. 41 (2014) 3004-3010.

[14] D. Güttler, F. Adolphi, J. Beer, N. Bleicher, G. Boswijk, M. Christl, A. Hogg, J. Palmer, C. Vockenhuber, L. Wacker, and J. Wunder, Rapid increase in cosmogenic ${ }^{14} C$ in $A D 775$ measured in New Zealand kauri trees indicates short-lived increase in ${ }^{14} C$ production spanning both hemispheres, Earth Planet. Sci. Lett. 411 (2015) 290-297.

[15] A. K. Pavlov, A. V. Blinov, A. N. Konstantinov, V. M. Ostryakov, G. I. Vasilyev, M. A. Vdovina, and P. A. Volkov, AD 775 pulse of cosmogenic radionuclides production as imprint of a Galactic gamma-ray burst, Mon. Notes R. Astron. Soc. 435 (2013) 2878-2884.

[16] E. W. Cliver, A. J. Tylka, W. F. Dietrich, and A. G. Ling, On a Solar Origin for the Cosmogenic Nuclide Event of 775 A.D., Astrophys. J. 781 (2014) 32. 
[17] J. Beer, K. McCracken, and R. von Steiger, Cosmogenic Radionuclides: Theory and Applications in the Terrestrial and Space Environments. Springer, Berlin, 2012.

[18] A. Tylka and W. Dietrich, A new and comprehensive analysis of proton spectra in ground-level encahnced (gle) solar particle events, in 31th International Cosmic Ray Conference, (Lodź, Poland), Universal Academy Press, 2009.

[19] G. A. Kovaltsov, I. G. Usoskin, E. W. Cliver, W. F. Dietrich, and A. J. Tylka, Fluence Ordering of Solar Energetic Proton Events Using Cosmogenic Radionuclide Data, Solar Phys. 289 (2014) 4691-4700.

[20] G. Kovaltsov, A. Mishev, and I. Usoskin, A new model of cosmogenic production of radiocarbon ${ }^{14} C$ in the atmosphere, Earth Planet. Sci. Lett. 337 (2012) 114-120.

[21] E. W. Cliver and W. F. Dietrich, The 1859 space weather event revisited: limits of extreme activity, J. Space Weath. Space Clim. 3 (2013), no. 26 A31.

[22] M. Stuiver, P. Reimer, E. Bard, G. Burr, K. Hughen, B. Kromer, G. McCormac, J. v.d. Plicht, and M. Spurk, Intcal98 radiocarbon age calibration, 24,000-0 cal bp, Radiocarbon 40 (1998), no. 3 1041-1083.

[23] F. Miyake, K. Masuda, and T. Nakamura, Another rapid event in the carbon-14 content of tree rings, Nature Comm. 4 (2013) 1748.

[24] G. A. Kovaltsov and I. G. Usoskin, A new 3D numerical model of cosmogenic nuclide ${ }^{10}$ Be production in the atmosphere, Earth Planet. Sci. Lett. 291 (2010) 182-188.

[25] K. McCracken, Geomagnetic and atmospheric effects upon the cosmogenic ${ }^{10}$ be observed in polar ice, J. Geophys. Res. 109 (2004), no. A18.

[26] I. G. Usoskin, K. Horiuchi, S. Solanki, G. A. Kovaltsov, and E. Bard, On the common solar signal in different cosmogenic isotope data sets, J. Geophys. Res. 114 (2009) A03112.

[27] H. S. Hudson, Solar flares add up, Nature Phys. 6 (2010), no. 9 637-638. 